\title{
Degenerative disease of the lumbar spine
}

\author{
Paul R. Cooper, M.D., ANd Anthony Frempong-Boadu, M.D. \\ Department of Neurosurgery, New York University Medical Center, New York, New York
}

In the past 15 years there has been an explosion of technical advances in the management of degenerative disease of the lumbar spine. From a conceptual point of view it has become apparent that in certain circumstances augmentation of pedicle screw fixation with transforaminal interbody fusion of the lumbar spine produces results that are superior to pedicle screw fixation alone in terms of success of the operation and patient satisfaction. Intraoperative image guidance has made the placement of lumbar instrumentation safer and more accurate. In the final analysis, the development of less invasive approaches to decompression and fusion of the lumbar spine has resulted in less postoperative pain, shorter hospital stays, decreased costs, and better preservation of the paraspinous musculature.
In this issue of Neurosurgical Focus, specific aspects of these advances are discussed. Minimally invasive surgical techniques for the excision of lumbar synovial cysts as well as for the placement of pedicle screws and the performance of transforaminal lumbar interbody fusion are described. The use of new and conventional intraoperative imaging is integral to the procedures detailed in several of these articles. The technical note on the management of high-grade spondylolisthesis represents a novel approach to a difficult problem.

The advances described in most of these articles would not have been imagined 25 years ago, and it is likely that 10 or 15 years from now, technical and conceptual advances will be even more far-reaching. We live in exciting times. 